\title{
Benefits of Prior Authorizations
}

\author{
Caroline Forrester, PharmD
}

Forrester's Viewpoint

$\mathrm{P}$ rior authorization is one of the most common drug utilization management tools in the U.S. health care system. The intent of prior authorizations is to ensure that drug therapy is medically necessary, clinically appropriate, and aligns with evidence-based guidelines. Although prior authorizations can be used to manage prescription drug costs, they may also improve patient outcomes by ensuring appropriate use and minimizing harmful drug interactions, adverse events, and/or off-label use for diagnoses that lack evidence to support use.

The benefits of prior authorizations include the following:

1. Management of prescription drug spend. Approximately $\$ 330$ billion was spent on prescription drugs in 2017, and the Centers for Medicare \& Medicaid Services projects that spending for retail prescription drugs will be the fastest growth health category and is projected to exceed $\$ 600$ billion by 2027. ${ }^{1}$ Consequently, payers use a variety of tools to help manage their prescription drug costs, one of which is prior authorization.

That some drugs may require a prior authorization to ensure they are medically necessary before approval of coverage may seem counterintuitive. By definition, if a physician prescribes a drug does that not mean it is medically necessary? In some cases, however, this may not necessarily be so, for example, (a) a prescription may be written for a costly brand name drug that has a therapeutically equivalent generic version available; (b) a prescription may be written for a costly corticosteroid cream, while a lower cost equal-potency cream is available; and (c) a prescription may be written for a costly tablet, while a lowercost capsule of the same medication is available.

In such cases, payers may implement prior authorizations on the costly medications. When prescribers who may not have been initially aware of the available alternatives are provided with this information, they may determine that the alternative is clinically appropriate for their patients and opt to switch. Thus, prior authorizations shift utilization to lower-cost medications in cases where the higher-cost versions provide no additional safety and/or efficacy benefits. There may be instances in which switching to a lower-cost alternative would not be clinically appropriate. Examples include efficacy and safety concerns, contraindications, allergies, or other patient-specific factors. Coverage may be approved once the prescriber provides information to support medical necessity of the originally prescribed medication. If coverage is not approved, the patient always has the option of obtaining the prescribed medication by paying out of pocket.

2. Clinically appropriate drug selection and utilization. To obtain approval by the U.S. Food and Drug Administration (FDA), a drug undergoes an approval process to ensure that it provides benefits for the target condition and population that outweigh its potential risks. ${ }^{2}$ Prior authorizations ensure that a drug is prescribed for patients with the specific FDA-approved indication. In cases where a drug is prescribed for off-label use, the prior authorization may be approved if it is prescribed for an indication that is supported by recognized compendia such as the American Hospital Formulary Service Drug Information (AHFS-DI), Micromedex DRUGDEX information system, LexiDrugs, Clinical Pharmacology, and the NCCN Drugs \& Biologics Compendium. Off-label indications may also be considered if supported by peer-reviewed medical literature, recognized standard of care, and/or recommended by national standard treatment guidelines. Similarly, prior authorizations may restrict drugs from use by patient populations for whom drugs are contraindicated. For example, Xgeva (denosumab) can cause severe symptomatic hypocalcemia, and fatal cases have been reported. Thus, it is contraindicated in patients with hypocalcemia. Prior authorization criteria may require documentation that the patient does not have hypocalcemia before approval.

The goal of prior authorizations is not to restrict appropriate use but rather to promote use in the patient population for whom safety and efficacy has been established. Furthermore, prior authorizations help to promote drug use that aligns with evidence-based and established clinical guidelines by requiring patients to first use drugs with more favorable safety and efficacy profiles and/or those that are preferred or recommended by treatment guidelines.

3. Promotion of safe drug use. Prescription drug abuse places a major health and economic burden on the U.S. health care system. Opioids are one of the most commonly misused drugs, 
with an estimated 3.2 million misusers reported in 2017. Approximately $50 \%$ of all opioid overdose deaths in the United States involve a prescription opioid, with about 17,000 cases resulting in death in 2017.,3

Prior authorizations may help curb this epidemic by providing opportunities for early intervention. For example, opioid prior authorizations may require submission of appropriate diagnoses or attestation from the prescriber in cases of duplicative opioid therapy or concurrent opioid and benzodiazepine therapy, especially in cases of multiple prescribers. A state Medicaid opioid prior authorization program composed of dosage limits and step therapy through lower-cost alternatives documented a decrease in overall opioid use and cost. ${ }^{5}$

4. To support drug formularies and rebate programs. Prior authorizations may be used to support use of preferred drugs. These drugs may be preferred because of cost differences, efficacy and/or safety differences, rebate programs, or alignment with evidence-based treatment guidelines. Formulary placement of a drug dictates the level of coverage-obtaining a formulary (vs. a nonformulary or excluded) drug results in lower out-of-pocket costs for a patient, thus shifting use towards preferred drugs. Supporting drug formularies and rebate programs help to decrease premium costs and/or cost-sharing payments for members.

\section{Effect of Prior Authorizations on Prescribers and Patients}

Prior authorization barriers may occur as a result of limitations created by prior authorization processes and limited amount of time and resources for prior authorization processing. Approximately $70 \%$ of prior authorization requests are initiated at the pharmacy as a result of a rejected claim. ${ }^{6}$ In majority of cases, prescribers do not become aware that their prescribed drugs require prior authorizations until they are notified by their patients or the pharmacist.

Fortunately, prior authorization processes can be improved with an enhanced understanding of the rationale for prior authorizations, as well as implementation of the following proposed strategies:

1. Refer to the patient's insurance plan drug formulary before prescribing a costly drug. Formularies can often be accessed for free online, and they provide a drug's formulary status and whether a prior authorization is required. Although time constraints may be a concern, accessing this information beforehand saves time, if the drug has formulary restrictions.

2. Consider using electronic prescribing and/or prior authorization systems that provide real-time access to drug benefit coverage information. Such automated systems allow prescribers to initiate prior authorization requests during prescribing, allowing automatic and timely review, since no paperwork or additional time to call or fax the patient's insurance company is required. Since the prior authorization is completed at the point of prescribing, joint treatment decisions can be made with the patient during their appointment. ${ }^{7-10}$ Challenges to electronic prescribing and prior authorizations include the need for investment in infrastructure, adaptation to changes in workflow, and payer support.

3. Respond to requests for information from the insurance company in a timely manner.

4. Ensure that the submitted diagnosis matches the drug. If submitting a request for off-label diagnosis, verify that the off-label diagnosis has compendia support or is accompanied by peer-reviewed literature sources showing established efficacy for the patient's diagnosis. If e-prescribing, diagnoses should be included if required. If prescribing for off-label use, the review will be based on compendia sources previously discussed.

5. Educate patients regarding their rights to initiate their own prior authorization requests. This would reduce the workload on prescribers and potentially reduce the chances of delays in therapy. Patients have a right to submit an appeal for a denied prior authorization.

I agree with Dr. William Bennett's statement in the accompanying viewpoint article that prescribers know their patients best, and payers only have the information provided to make a decision. Prior authorization denials can occur as a result of prescribers not responding to requests for additional information to support approval. This is why the information submitted is essential to the outcome of the prior authorization. In Bennett's example of prescribing a biologic that is not approved by the FDA in pediatric patients, the payer is essentially seeking supporting evidence. In the absence of FDA approval and randomized controlled trials, submission of any supporting documentation, such as observational studies, case control studies, cross-sectional studies, treatment guidelines, and/or consensus statements is beneficial. Payers have criteria for what information they would consider; however, in cases such as these, the decision will be based on the clinical judgment of the reviewer, so providing as much information as necessary to support a favorable determination is advantageous. Other strategies include proactive submission of prior authorization (well in advance of needing to initiate therapy) and requesting urgent review to reduce turnaround time.

\section{Summary}

Prior authorization criteria are based on scientific evidence, standards of practice, peer-reviewed medical literature, established clinical practice guidelines, as well as safety and efficacy data. The goal of prior authorizations is not to create undue burden on patients or to intentionally prevent access. Prior authorization barriers can be mitigated by a better understanding of the rationale for, and benefits of, prior authorizations. 


\section{Author}

CAROLINE FORRESTER, PharmD, MedImpact Healthcare Systems, San Diego, California.

AUTHOR CORRESPONDENCE: Caroline Forrester, PharmD, MedImpact Healthcare Systems, 10181 Scripps Gateway Ct., San Diego, CA 92131.E-mail: caroline.forrester@medimpact.com.

\section{DISCLOSURES}

No funding supported the writing of this article. The author has nothing to disclose.

\section{REFERENCES}

1. Centers for Medicare \& Medicaid Services. National health expenditure projections 2018-2027. Available at: https://www.cms. gov/ Research-Statistics-Data-and-Systems/Statistics-Trends-and-Reports/ NationalHealthExpendData/NationalHealthAccountsProjected. Accessed May 17, 2020.

2. U.S. Food and Drug Administration. Drug development and approval process. Available at: https://www.fda.gov/drugs/development-approval-processdrugs\#FDA. Accessed May 17, 2020.

3. Substance Abuse and Mental Health Services Administration. Key substance use and mental health indicators in the United States: results from the 2017 National Survey on Drug Use and Health. HHS Publication no. SMA 18-5068, NSDUH Series H-53. September 2018. Available at: https:// www.samhsa.gov/data/report/2017-nsduh-annual-national-report. Accessed May 17, 2020.
4. Centers for Disease Control and Prevention. Opioid overdose: Rx Awareness. Updated October 24, 2017. Available at: https://www.cdc.gov/ rxawareness/about/index.html. Accessed May 17, 2020.

5. Garcia MM, Angelini MC, Thomas T, et al. Implementation of an opioid management initiative by a state Medicaid program. J Manag Care Spec Pharm. 2014;20(5):447-54. Available at: https://www.jmcp.org/doi/ pdf/10.18553/jmcp.2014.20.5.447. Accessed May 17, 2020.

6. Hessick J. Electronic prior authorization can increase dispensing workflow of specialty medications. Pharmacy Times. August 16, 2016. Available at: https://www.specialtypharmacytimes.com/publications/specialty-pharmacy-times/2016/July-2016/Electronic-Prior-Authorization-can-IncreaseDispensing-Workflow-of-Specialty-Medications. Accessed May 17, 2020.

7. CoverMyMeds. 2020 electronic prior authorization report. Available at: https://assets.ctfassets.net/2in405srp47m/4uzia8m4YQy3mBplpyBuqF/9af 9cefa63bba76566aa3e859575le45/CMM_36664_ePAExecutiveSummary_ Digital.pdf. Accessed May 31, 2020.

8. Centers for Medicare \& Medicaid Services. E-prescribing. Updated February 11, 2020. Available at: https://www.cms.gov/Medicare/E-Health/ Eprescribing/index.html?redirect=/eprescribing. Accessed May 17, 2020.

9. Electronic prescribing: the first step towards an integrated health delivery system? The Rheumatologist. February 1, 2009. Available at: https://www. the-rheumatologist.org/article/electronic-prescribing-the-first-step-towards-anintegrated-health-delivery-system/. Accessed May 31, 2020.

10. Salmon JW, Jiang R. E-prescribing: history, issues, and potentials. Online J Public Health Inform. 2012;4(3):el0.

\title{
Insurance Denials of Care Amount to Unlicensed Medical Practice
}

\author{
William E. Bennett, MD, MS
}

\section{Bennett's Viewpoint}

$\Lambda$ s a specialist who often orders expensive medications, I find a significant portion of my week is spent addressing denials from insurance companies. Our team has extensive experience caring for complex, severely ill patients. We make recommendations after careful

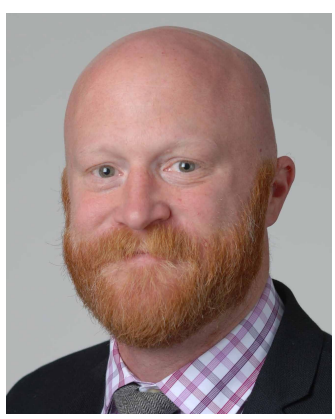

consideration of the risks and benefits, examining the patient, reviewing the chart in detail, and meeting with the family to understand their preferences. Then, our recommendation is countered by some sort of denial—either an outright denial or a request for prior authorization, which usually amounts to a denial with the opportunity to appeal. This practice by payers is directly harmful to patients, and the health care system, because it (a) amounts to an unqualified entity practicing medicine with limited information about the patient, (b) does not actually contain costs, and (c) is not an effective method to improve care quality. 